\title{
La Taxonomía de Bloom para la era digital: actividades digitales docentes en octavo, noveno y décimo grado de Educación General Básica (EGB) en la Habilidad de "Comprender»
}

\author{
Bloom's Taxonomy for the Digital Age: eighth, ninth, and tenth grade digital activities for \\ Basic General Education (EGB) in the Ability to "Understand»
}

\author{
CUENCA, Alex A. ${ }^{1}$ \\ ALVAREZ, Mauricio. ${ }^{2}$ \\ ONTANEDA, Luis J. ${ }^{3}$ \\ ONTANEDA, Elvis A. ${ }^{4}$ \\ ONTANEDA, Sybel E. ${ }^{5}$
}

\begin{abstract}
Resumen
Actualmente predomina la tecnología digital y motiva a determinar, a través de una metodología científica de tipo descriptiva - correlacional, la aplicabilidad de la Taxonomía de Bloom, mediante actividades digitales docentes en Instituciones educativas de tipo Fiscal, Fiscomisional y Particular, para potenciar el nivel de comprensión en los estudiantes de Octavo, Noveno y Décimo Grado de Educación General Básica (EGB), durante el año lectivo 2019-2020, en el sector urbano de la ciudad de Loja Ecuador, como referente de modernización, donde se logró evidenciar mayor aplicabilidad en la Institución Particular.

Palabras clave: taxonomía de bloom, actividades digitales, comprensión.

Abstract

Currently digital technology predominates and motivates to determine through a descriptivecorrelational scientific methodology, the applicability of Bloom's Taxonomy, through digital teaching activities in educational institutions of Fiscal, Fiscomisional and Private type to enhance the level of understanding in students of Eighth, Ninth and Tenth Grade of Basic General Education (EGB), during the 2019-2020 school year in the urban sector of the city of Loja - Ecuador, as a benchmark of modernization, where it was possible to demonstrate greater applicability in the Private Institution. key words: bloom's taxonomy, digital activities, understanding.
\end{abstract}

\footnotetext{
${ }^{1}$ Docente. Instituto Superior Tecnológico "Los Andes". Ecuador. alexcuenca@institutolosandes.edu.ec

2 Docente. Universidad Católica de Cuenca. Ecuador. ideasgrupecuador@gmail.com

${ }^{3}$ Director Académico. Instituto Coach Group Ecuador. Ecuador. luisjavierov@gmail.com

${ }^{4}$ Director General. Instituto Coach Group Ecuador. Ecuador. eaontaneda@gmail.com

${ }^{5}$ Docente Investigador. Universidad Nacional de Loja. Ecuador. sybel.ontaneda@unl.edu.ec
} 


\section{Introducción}

En el año de 1956, Benjamín Bloom enunció una taxonomía denominada como Taxonomía de Bloom, con el propósito de facilitar a los docentes la evaluación del nivel cognitivo adquirido por los estudiantes en sus procesos de aprendizaje; dicha Taxonomía consta de niveles en el proceso de pensamiento, desde Habilidades de Pensamiento de Orden Inferior (LOTS): Conocimiento, Compresión, Aplicación; hasta Habilidades de Pensamiento de Orden Superior (HOTS): Análisis, Síntesis y Evaluación (Hallak , y otros, 2000; Churches, 2009). En 2001 Anderson y Krathwohl hicieron revisiones que constan de dos dimensiones: una para los procesos cognitivos y otra para el conocimiento, en el cual se efectúa la sustitución de los sustantivos por el uso de verbos; la modificación consiste en lo siguiente, en orden ascendente: recordar, comprender, aplicar, analizar, evaluar y crear. Posteriormente en el 2009, el Dr. Andrew Churches realizó una actualización de la Taxonomía Revisada de Bloom para la Era Digital introduciendo nuevas acciones coherentes con las tecnologías digitales actuales como ("realizar búsquedas en los navegadores", "subir archivos a la nube", "recopilar información de medios", "publicar", etc.).

La Taxonomía de Bloom en la Era Digital brinda muchos beneficios, por ejemplo ("uso de redes sociales favoreciendo la colaboración", "uso de editores de video para mejorar la presentación de un video"), por ser un marco referencial a nivel docente que permite fijar de forma clara y concisa los objetivos formativos o resultados de aprendizaje. Es así como en la era digital esta Taxonomía es empleada como referente para las recomendaciones curriculares para la elaboración de los objetivos de aprendizaje; además permite conocer y desarrollar diferentes procesos educativos y saber las capacidades adquiridas por los estudiantes, de manera que el CEAACES (Consejo de Evaluación Acreditación y Aseguramiento de la Educación Superior) en el Ecuador, sigue la Taxonomía de Bloom donde se distinguen tres tipos de resultados de aprendizaje, relacionados con el dominio cognitivo, el dominio de las aptitudes o habilidades y el de las actitudes (Der Bijl, 2015). Partiendo como base del proceso de evaluación en las carreras universitarias del Ecuador y la realidad de la Cuarta Revolución Industrial de la Era Digital, se ha logrado complementar cada categoría con verbos y herramientas del mundo virtual en esta Taxonomía, posibilitando el desarrollo de habilidades en los docentes que forman a los futuros estudiantes universitarios (Churches, 2009).

Como referente nacional universitario se debe aplicar la Taxonomía de Bloom mediante actividades digitales, manteniendo una relación directa con la estructuración de los resultados de aprendizaje según los sílabos universitarios, que atiende los nuevos procedimientos, acciones y oportunidades en el proceso educativo que surgen a medida que las TIC (Tecnologías de la Información y las Comunicación) avanzan y se vuelven más presentes en el sistema académico; por ello se ha establecido el objetivo de determinar la aplicabilidad de la Taxonomía de Bloom, mediante actividades digitales docentes en Octavo, Noveno y Décimo Grado de Educación General Básica para "Comprender"; recalcando que se debe iniciar el proceso desde el entendimiento para poder alcanzar una aplicabilidad efectiva en los docentes que no están capacitados para ejercer en el campo virtual.

\subsection{Problemática}

En la actualidad a nivel mundial en la sociedad del conocimiento donde estamos inmersos, especificamente en el contexto educativo ha desarrollado una dependencia tecnológica lo cual es una muestra clara que la educación debe estar a la vanguardia tecnológica, donde los docentes tienen que ser hábiles para manejar una serie de conceptos, habilidades y actitudes que les permitan enfrentarse a la resolución de problemas y a una toma de decisiones responsable y autónoma en donde el aprendizaje debe ser adquirido por los estudiantes, pero a la vez, comprendido para poder ser aplicado en la resolución de los problemas reales (Hargreaves, 2003). Ante ello se ha contemplado un déficit en cuanto a la formación de los docentes dentro de este ámbito, de tal forma que ante esta problemática se requiere que los docentes estén en constante actualización y capacitación desde las 
bases para que comprendan, aprendan, apliquen y puedan brindar a sus estudiantes una educación de calidad. Es así como ante esta necesidad se encuentra la Taxonomía de Bloom para la era digital, la cual es fundamental como un marco referencial a nivel docente que permite fijar de forma clara y concisa los objetivos formativos o resultados de aprendizaje relevantes a nivel curricular que permite evaluar el nivel cognitivo por cada área de estudio, respetando los niveles de aprendizaje (Chero, 2009; Eduteka, 2010; Van Der Bijl, 2015).

\subsection{Revisión de literatura}

\subsubsection{La Taxonomía de Bloom}

La Taxonomía de Bloom es un marco referencial para los docentes los cuales la consideran ideal para la evaluación del nivel cognitivo adquirido en una determinada área de conocimiento, por ejemplo, al aplicar conceptos adquiridos se requiere en los niveles de la taxonomía, recordar y entender (Masapanta y Velázquez, 2018; Churches, 2009; Fuller, y otros, 2007; Bloom, 1990).

Fundamentalmente, a través de esta Taxonomía los estudiantes adquieren nuevas habilidades y conocimientos, por tal razón se presentan varios niveles cada uno con el propósito de garantizar a los docentes el idóneo aprendizaje de los estudiantes (Masapanta y Velázquez, 2018; Churches, 2009). La Taxonomía y sus variaciones han apoyado a los docentes en su pensamiento o el dar respuesta a la importante cuestión de como aprenden los estudiantes y que situación atender en las programaciones del aula (Careiro, 2019; Hallak , y otros, 2000; Woolfolk, 2010).

\section{Niveles de la Taxonomía de Bloom}

La taxonomía cognitiva de Bloom contiene seis niveles de complejidad creciente los cuales son: Conocimiento, Comprensión, Aplicación, Análisis, Síntesis y Evaluación con subniveles identificados. El desempeño en cada nivel depende del estudiante en el nivel o los niveles precedentes. Por ejemplo, para la evaluación del nivel más alto el estudiante debe estar en capacidad de que comprenda, aplique, analice, sintetice y finalmente evalué la información adquirida (Barcia Menéndez, Carvajal Zambrano, Barcia Menéndez, y Sánchez García, 2017). Como se puede determinar, en base al ejemplo anterior, los diferentes niveles se encuentran en orden jerárquico de acuerdo con los procesos cognitivos (Fuller, y otros, 2007).

La Taxonomía de Bloom ordena un conjunto de habilidades intelectuales desde un nivel simple hacia la complejidad. Además de ser jerárquico es acumulativo, puesto que para avanzar un nivel el estudiante tiene que dominar el nivel anterior (Barcia Menéndez, Carvajal Zambrano, Barcia Menéndez, y Sánchez García, 2017).

En 1956 Benjamín Bloom examinó, a partir del dominio cognitivo, habilidades de pensamiento y objetivos, las mismas que fueron categorizadas y ordenadas; su propuesta va en orden ascendente desde Habilidades de Pensamiento de Orden Inferior (LOTS) hasta las Habilidades de Pensamiento de Orden Superior (HOTS). En el mismo, cada nivel se encuentra descrito por sustantivos (López, 2014; Forehand, 2011; Geoff, 1996; Hyder y Bhamani, 2016; Aliaga, 2012) (Tabla 1).

Posterior a ello, la Taxonomía de Bloom es revisada, en el 2001, por Lorin Anderson, quien la publicó como Taxonomía Revisada de Bloom. La nueva publicación hace uso de verbos a diferencia de la anterior que empleaba sustantivos por cada categoría. Otro de los cambios es la secuencia; este cambio considera la creatividad como superior a la evaluación dentro del dominio cognitivo (Churches, 2009) (Tabla 2). 
Tabla 1

Taxonomía de Bloom

\begin{tabular}{|c|c|c|}
\hline \multicolumn{2}{|c|}{$\begin{array}{c}\text { Habilidades de Pensamiento } \\
\text { de Orden Superior (HOTS) }\end{array}$} & Descripción \\
\hline Evaluación & & $\begin{array}{l}\text { Habilidad para obtener juicios sobre el valor para ideas, } \\
\text { trabajos, materiales, soluciones, métodos. Pueden ser } \\
\text { cuantitativos y/o cualitativas. La evaluación se efectúa con } \\
\text { respecto a criterios internos y/o externos. }\end{array}$ \\
\hline Síntesis & & $\begin{array}{c}\text { Habilidad para agrupar elementos y partes para elaborar un } \\
\text { todo nuevo, con énfasis de crear, a fin de elaborar un patrón o } \\
\text { estructura que no se especifica. }\end{array}$ \\
\hline Análisis & & $\begin{array}{l}\text { Habilidad que enfatiza en la descomposición de una o todas sus } \\
\text { partes constituyentes. }\end{array}$ \\
\hline Aplicación & & $\begin{array}{c}\text { Utilizar comprensiones logradas, para resolver un problema real } \\
\text { o ideal, planteando términos científicos de forma relativa al } \\
\text { fenómeno pertinente. }\end{array}$ \\
\hline Comprensión & & $\begin{array}{l}\text { Comprender una comunicación, al lograrlo, el estudiante puede } \\
\text { cambiar la comunicación para darle un mejor significado. }\end{array}$ \\
\hline Conocimiento & & $\begin{array}{l}\text { Memorizar información que se expresa por "Reconocimiento, } \\
\text { recuerdo", de hechos específicos aislados. }\end{array}$ \\
\hline $\begin{array}{l}\text { Habilidades de } \\
\text { de Orden Infe }\end{array}$ & $\begin{array}{l}\text { Pensamiento } \\
\text { erior (LOTS) }\end{array}$ & \\
\hline
\end{tabular}

Fuente: Aliaga (2012)

Tabla 2

Taxonomía Revisada de Bloom, niveles y verbos

\begin{tabular}{|c|c|}
\hline $\begin{array}{l}\text { Habilidades de } \\
\text { de Orden Sup }\end{array}$ & Verbos \\
\hline Crear & Diseñar, construir, planear, producir, idear, trazar, elaborar. \\
\hline Evaluar & $\begin{array}{l}\text { Revisar, formular hipótesis, criticar, experimentar, juzgar, } \\
\text { probar, detectar, monitorear. }\end{array}$ \\
\hline \multirow{2}{*}{ Analizar } & Comparar, organizar, deconstruir, atribuir, delinear, \\
\hline & encontrar, estructurar, integrar. \\
\hline Aplicar & Implementar, despeñar, usar, ejecutar. \\
\hline Comprender & $\begin{array}{l}\text { Interpretar, resumir, inferir, parafrasear, clasificar, comparar, } \\
\text { explicar, ejemplificar. }\end{array}$ \\
\hline Recordar & $\begin{array}{l}\text { Reconocer, listar, describir, identificar, recuperar, denominar, } \\
\text { localizar, encontrar. }\end{array}$ \\
\hline $\begin{array}{l}\text { Habilidades de Pensamiento } \\
\text { de Orden Inferior (LOTS) }\end{array}$ & \\
\hline
\end{tabular}

Fuente: Amor Pérez y Delgado Huelva, 2012; Churches (2009) 


\section{La Taxonomía de Bloom en la era digital}

La Taxonomía de Bloom a lo largo de los años ha continuado siendo una herramienta esencial para el establecimiento de objetivos de aprendizaje, de tal forma que ha sufrido cambios con el propósito de atender los nuevos objetivos, procesos y acciones para las prácticas actuales en la que se incluyen las TIC (Amor Pérez y Delgado Huelva, 2012; Churches, 2009; Hyder y Bhamani, 2016).

Churches (2009), citado por Amor Pérez y Delgado Huelva (2012), quien actualizó la taxonomía y la puso en relación con las nuevas realidades de la era digital, complementó cada categoría con verbos y herramientas del mundo digital, todo ello con el fin de desarrollar habilidades tales como recordar, comprender, aplicar, analizar, evaluar y crear (Tabla 3). La adaptación de Churches (2009) establece una relación de los verbos que describen las habilidades de pensamiento con el lenguaje técnico informático producido en la comunicación debido a los cambios tecnológicos.

Tabla 3

Taxonomía Digital de Bloom

\begin{tabular}{|c|c|c|}
\hline $\begin{array}{l}\text { Habilidades d } \\
\text { de Orden Sup }\end{array}$ & Verbos de entorno digital & Actividades \\
\hline Crear & $\begin{array}{l}\text { Programar, filmar, animas, bloguear, video } \\
\text { bloguear, mezclar, participar en un wiki, publicar, } \\
\text { videocasting, dirigir, transmitir. }\end{array}$ & $\begin{array}{l}\text { Colaborar } \\
\text { Moderar } \\
\text { Negociar }\end{array}$ \\
\hline Evaluar & $\begin{array}{c}\text { Comentar en un blog, revisar, publicar, moderar, } \\
\text { colaborar, participar en redes (networking), } \\
\text { reelaborar, probar. }\end{array}$ & $\begin{array}{c}\text { Debatir } \\
\text { Comentar } \\
\text { Reunirse en la red }\end{array}$ \\
\hline Analizar & $\begin{array}{l}\text { Recombinar, enlazar, validar, hacer ingeniería } \\
\text { inversa, craking, recopilar información de medios. }\end{array}$ & $\begin{array}{c}\text { Realizar videoconferencias } \\
\text { por Skype } \\
\text { Revisar }\end{array}$ \\
\hline Aplicar & $\begin{array}{l}\text { Correr, cargar, jugar, operar, hackear, subir } \\
\text { archivos a un servidor, compartir, editar. }\end{array}$ & $\begin{array}{l}\text { Preguntar } \\
\text { Contestar }\end{array}$ \\
\hline Comprender & $\begin{array}{c}\text { Hacer búsquedas avanzadas, hacer búsquedas } \\
\text { Booleanas, hacer periodismo en formato blog, usas } \\
\text { Twitter, categorizar, etiquetar, comentar, anotar, } \\
\text { suscribir. }\end{array}$ & $\begin{array}{l}\text { Publicar y blogear } \\
\text { Participar en redes } \\
\text { Contribuir } \\
\text { Chatear }\end{array}$ \\
\hline Recordar & $\begin{array}{c}\text { Utilizar viñetas, resaltar, marcar, participar en la } \\
\text { red social, marcar sitios favoritos, buscar, hacer } \\
\text { búsquedas en Google. }\end{array}$ & $\begin{array}{l}\text { Comunicarse por correo } \\
\text { electrónico } \\
\text { Comunicarse por Twitter/ }\end{array}$ \\
\hline \begin{tabular}{|l|}
$\begin{array}{l}\text { Habilidades de Pensamiento } \\
\text { de Nivel Inferior }\end{array}$ \\
\end{tabular} & & $\begin{array}{c}\text { Microblogs } \\
\text { Mensajería instantánea }\end{array}$ \\
\hline
\end{tabular}

Fuente: Amor Pérez y Delgado Huelva, 2012; Churches, 2009; Aliaga, 2012.

Autor: Elaboración propia

\section{Nivel de Comprender}

El comprender constituye relaciones y une conocimientos. En este nivel los estudiantes comprenden procesos y conceptos, pueden resumirlos y parafrasearlos con sus propias palabras, de tal forma que puedan establecer relaciones y construir significados. La construcción de significados puede efectuarse a través de funciones escritas o gráficas (Amor Pérez y Delgado Huelva, 2012; Churches, 2009).

Las adiciones digitales para efectuarse en este nivel son las siguientes (Amor Pérez y Delgado Huelva, 2012; Churches, 2009):

- Búsqueda Avanzada y Booleana: Los estudiantes deben comprender de forma insondable para así poder crear, modificar y refinar búsquedas que se adapten a sus necesidades. 
- Periodismo en formato blog (Blog Journalling): En un blog el estudiante habla, escribe o digita un diario personal o sobre una tarea específica, esto destaca la compresión básica de la actividad que se está efectuando.

- Categorizar y Etiquetar: Organizar y clasificar archivos digitales, sitios web y materiales, empleando folders, delicius y otras herramientas análogas; para ir más allá los estudiantes requieren ir organizando, estructurando y asignando datos en línea, palabras claves en el encabezado de páginas Web, al comprender el contenido de las páginas los estudiantes podrán etiquetarlas.

- Comentar y anotar: Existen varias herramientas que permiten al estudiante comentar y efectuar anotaciones en páginas Web, pdf, entradas en marcadores sociales y otros documentos, el estudiante comprende a través de efectuar comentarios sobre las páginas.

- Suscribir: Suscribirse al servicio RSS de un sitio emplea la marcación en sus diversas formas y eleva de nivel la lectura, el acto de leer y revisar nuevamente lo publicado en las entradas conduce a una mayor compresión.

\section{Actividades digitales para Comprender}

Las actividades a llevarse a cabo para que los estudiantes comprendan son las siguientes (Amor Pérez y Delgado Huelva, 2012; Churches, 2009):

- Resumir: Se puede efectuar a través de procesadores de texto, mapas conceptuales, publicar en la Web, Auto publicaciones simples (diarios en blogs y paginas sencillas de elaboración colaborativa de documentos).

- Recolectar: Procesador de texto, mapa mental, publicar en la Web, diarios en blogs y páginas sencillas de construcción colaborativa de documentos, Wiki.

- Explicar: Procesador de texto, mapas conceptuales, publicar en la Web, Auto publicaciones simples, Wikis.

- Mostrar y contar: Procesador de texto, presentaciones en línea o locales, gráficas, herramientas de audio, herramientas de video, mapa mental.

- Listar: Procesador de texto, mapas conceptuales.

- Etiquetar: Procesador de texto, mapas conceptuales, gráficas, herramientas en línea (Ajaxdraw).

- Bosquejar: Procesador de texto, mapa mental.

- Hacer Búsquedas avanzadas y Booleanas: Funciones avanzadas de búsqueda (Google, Explorer, Firefox entre otros).

- Alimentar un diario en Blog: Bloglines, Blogger, WordPress, entre otros.

- Publicar a diario: Blogging, Myspaces, Bebo, Facebook, Bloglines, Blogger, Ning, Twitter.

- Categorizar y etiquetar: Delicious, entre otros.

- Etiquetar, registrar comentarios: Noticeboards, foros de discusión, discusiones en cadena, lectores de archivos PDF, Blogs, Firefox, Zotero.

- Suscribir: Agregadores RSS (Bloglines, Google Reader entre otros), extensiones de Firefox.

\subsection{Justificación}

La razón del presente estudio de aplicación docente de la Taxonomía de Bloom para la Era Digital enfocada directamente en el la Habilidad "Comprender", requiere un análisis a nivel de pensamiento sobre que conocimientos han adquirido los docentes, resultando esencial que se emplee actividades digitales para contribuir en la comprensión de los estudiantes de los diferentes contenidos de información, así también es primordial para los docentes conocer el escenario en el que se encuentra y efectuar cambios en las actividades del aula. 
Ante esta situación el presente estudio analiza las actividades digitales en el nivel de Educación General Básica en diferentes Instituciones tanto como Fiscal, Fiscomisional y de índole Particular, en la Zona 7 esencialmente en la zona urbana de la ciudad de Loja, en el periodo 2019 - 2020, aclarando que los centros seleccionados cuentan con al menos un laboratorio de computación y también acceden a las plataformas digitales del Ministerio de Educación del Ecuador como la Plataforma de Gestión Educar Ecuador (https://educarecuador.gob.ec/sistemade-gestion-de-control-escolar/), resultando primordial las competencias digitales docentes como parte de la automatización de los servicios educativos.

\subsection{Objetivo}

Determinar la aplicabilidad de la Taxonomía de Bloom, mediante actividades digitales docentes en instituciones educativas de tipo Fiscal, Fiscomisional y Particular para potenciar el nivel de comprensión en los estudiantes de EGB durante el año lectivo 2019-2020 en el sector urbano de la ciudad de Loja - Ecuador.

\section{Metodología}

\subsection{Método}

El presente estudio utiliza un método descriptivo - correlacional. Según Cazau (2006), un estudio descriptivo constituye puramente una descripción de algunos fenómenos, situaciones, contextos y sucesos, siendo su objetivo esencial la medición de una o más variables dependientes, en alguna población definida o en una muestra de esta, es decir, sirve para describir cómo es y se manifiesta un fenómeno y sus componentes.

Adicional a ello cabe citar a los autores Hernández Sampieri, Fernández Collado y Baptista Lucio (2014), quienes afirman que se precisa llevar a cabo estudios de correlación para analizar el comportamiento de un concepto o variable en relación con otras variables vinculadas; lo que nos permite determinar la aplicabilidad de la Taxonomía de Bloom en los tres niveles de educación Fiscal, Fiscomisional y Particular con respecto a la habilidad de Comprender, como base de la era digital.

\subsection{Descripción del contexto}

El estudio se enfoca en tres instituciones educativas ubicadas al Sur del Ecuador, región Sierra, en el Circuito de la Parroquia San Sebastián (INEC 110102), correspondiente al Distrito 11D01 en la Zona 7 de Educación, de tipo Fiscal, Fiscomisional y Particular, pertenecientes al sector urbano de la ciudad de Loja; aclarando que se establece una comparativa entre los tipos de centros antes mencionados; contando con la participación directa de un total de 63 docentes durante el periodo lectivo 2019 - 2020; que brindaron información relevante para determinar la aplicabilidad de la Taxonomía de Bloom, para potenciar el nivel de Comprensión.

\subsection{Instrumento}

EL presente estudio utiliza un cuestionario ad hoc denominado "Aplicabilidad de la Taxonomía de Bloom, mediante actividades digitales docentes en instituciones educativas para potenciar el nivel de comprensión en los estudiantes de EGB", basado en Churches (2009), qué contiene 5 dimensiones de las Actividades Digitales para Comprender, con 3 ítems para los tipos de institución educativa, Fiscal, Fiscomisional y Particular.

Como su nombre lo indica el cuestionario se encuentra estructurado con el propósito de determinar la aplicabilidad del referente teórico "Taxonomía de Bloom", a través de las diferentes actividades digitales de los docentes de las tres Instituciones Educativas involucradas, para tener de base un referente que permite potenciar el nivel de Comprensión en los estudiantes de EGB durante el año lectivo 2019-2020 en el sector urbano de la ciudad de Loja - Ecuador. 


\subsection{Procedimiento}

Al inicio del estudio se eligió la Zona 7, particularmente la parte urbana de la ciudad de Loja, seleccionando posteriormente instituciones educativas de carácter Fiscal, Fiscomisional y Particular, luego se realizaron solicitudes por escrito a las autoridades de las instituciones educativas en este caso a los Rectores, requiriendo el permiso respectivo para la aplicación en línea mediante Google Forms de un cuestionario ad hoc a los docentes, una vez obtenida la aceptación correspondiente, se organizó y procesó la información, para luego analizar e interpretarla.

\section{Resultados y discusión}

Luego de haber obtenido los datos, se analiza la aplicación de la habilidad de Comprender en la Taxonomía de Bloom para la era digital y las cinco actividades que esta implica a nivel docente, estableciendo el nivel porcentual con respecto a los tres tipos de institución que participaron en la investigación, concordando con los estándares de calidad educativa a nivel de país, promulgados por el Ministerio de Educación del Ecuador en el año 2012 y la actualización del 2017.

\subsection{Actividad Digital para Comprender: Resumir}

Tabla 4

Actividad digital para Comprender: Resumir

\begin{tabular}{|l|r|}
\hline \multicolumn{2}{|c|}{$\begin{array}{c}\text { Actividad Digital en la Habilidad Comprender: Actividad } \\
\text { Resumir }\end{array}$} \\
\hline Institución Educativa Fiscal & $12,12 \%$ \\
\hline Institución Educativa Fiscomisional & $35,11 \%$ \\
\hline Institución Educativa Particular & $52,77 \%$ \\
\hline TOTAL & $100,00 \%$ \\
\hline
\end{tabular}

Fuente: Encuesta La Taxonomía de Bloom para la Era Digital

En la Tabla 4 se refleja los resultados obtenidos por los docentes en la utilización de la actividad digital de la Taxonomía de Bloom en la habilidad Comprender y en la actividad de Resumir, la misma que cosiste en un proceso de identificar ideas importantes en un determinado orden (Amor Pérez y Delgado Huelva, 2012; Anderson y Krathwohl, 2001; Churches, 2009), teniéndose así como resultado que la Institución Educativa Particular obtuvo el mayor porcentaje de aplicación docente con 52,77\%, cuenta con docentes que consideran en su mayoría aplicar las actividades digitales para comprender mediante la actividad de resumir, seguida de la institución Fiscomisional con el 35,11\% y finalmente la Institución Educativa Fiscal con el porcentaje mínimo del $12 \%$; reflejando que la primera institución educativa mencionada se encuentra en mejores condiciones para comprender la esencia de los contenidos curriculares adquiridos a través de actividades digitales tales como: procesadores de texto, mapas conceptuales, publicaciones en la Web, entre otros, según lo menciona El autor Churches (2009) citado por Amor Pérez y Delgado Huelva (2012). 


\subsection{Actividad Digital para Comprender: Recolectar}

Tabla 5

Actividad digital para Comprender: Recolectar

\begin{tabular}{|l|r|}
\hline \multicolumn{2}{|c|}{ Actividad Digital en la Habilidad Comprender: Actividad } \\
Recolectar \\
\hline Institución Educativa Fiscal & $26,98 \%$ \\
\hline Institución Educativa Fiscomisional & $40,87 \%$ \\
\hline Institución Educativa Particular & $32,15 \%$ \\
\hline TOTAL & $100,00 \%$ \\
\hline
\end{tabular}

Fuente: Encuesta La Taxonomía de Bloom para la Era Digital

De acuerdo a los datos de la Tabla 5 se puede evidenciar la actividad digital de la Taxonomía de Bloom en la habilidad Comprender y en la actividad de Recolectar que se refiere a reunir y medir información de diversas fuentes a fin de obtener un panorama completo y preciso de un cierto contexto (Amor Pérez y Delgado Huelva, 2012; Anderson y Krathwohl, 2001; Churches, 2009), con un $40.87 \%$ los docentes de la Institución Fiscomisional emplea positivamente esta habilidad y actividad, seguidamente se encuentra la Institución Educativa particular con el $32.15 \%$, y en menor proporción con un 26.98\% las Instituciones Educativas Fiscales.

Con esos resultados se evidencia que la Institución Educativa Fiscomisional es la que mayormente emplea la actividad para Recolectar, donde los docentes aplican las actividades como lo menciona el autor Churches (2009) citado por Amor Pérez y Delgado Huelva (2012), que consisten en procesar textos, organizadores gráficos, publicaciones Web, diarios en blogs y páginas sencillas de construcción colaborativa de documentos y Wikis.

\subsection{Actividad Digital para Comprender: Explicar}

Tabla 6

Actividad digital para Comprender: Explicar

\begin{tabular}{|l|r|}
\hline \multicolumn{2}{|c|}{ Actividad Digital en la Habilidad Comprender: Actividad } \\
Explicar \\
\hline Institución Educativa Fiscal & $20,14 \%$ \\
\hline Institución Educativa Fiscomisional & $39,02 \%$ \\
\hline Institución Educativa Particular & $40,84 \%$ \\
\hline TOTAL & $100,00 \%$ \\
\hline
\end{tabular}

Fuente: Encuesta La Taxonomía de Bloom para la Era Digital

Se puede evidenciar en la Tabla 6 los resultados del alcance de la actividad digital de la Taxonomía de Bloom en la habilidad Comprender y en la actividad de Explicar, la misma que consiste en la transmisión de conocimientos a terceros para que sean aprendidos y entendidos de forma clara y precisa (Amor Pérez y Delgado Huelva, 2012; Anderson y Krathwohl, 2001; Churches, 2009), según los datos obtenidos los docentes de la Institución Particular emplean mayormente la habilidad Comprender y la actividad de Explicar con un $40.84 \%$, los docentes de la Institución Fiscomisional ocupan un 39.02\% y en menor porcentaje los docentes de la Institución Fiscal con $20.14 \%$.

Esto indica que los docentes de la Institución Particular son quienes están más preparados para utilizar la actividad digital para Explicar, como procesadores de texto, mapas conceptuales, publicaciones en la Web, auto aplicaciones simples, Wikis, entre otros, a la par sirven de complemento a los estudiantes para que puedan 
"Explicar", lo comprendido referente a los contenidos de información que adquieran, coincidiendo con el criterio de Churches (2009), quien afirma que estas actividades ayudan a fortalecer la actividad de "Explicar" de una forma lúdica y motivacional en el aula y fuera de ella. Esto ayuda a potenciar las Habilidades de Pensamiento de Orden Superior (HOTS), ya que en cada nivel se encuentra descrito por sustantivos (López, 2014; Forehand, 2011; Geoff, 1996; Hyder y Bhamani, 2016; Aliaga, 2012).

\subsection{Actividad Digital para Comprender: Mostrar y Contar}

Tabla 7

Actividad digital para Comprender: Mostrar y Contar

\begin{tabular}{|l|r|}
\hline \multicolumn{2}{|c|}{ Actividad Digital en la Habilidad Comprender: Actividad } \\
Mostrar y Contar \\
\hline Institución Educativa Fiscal & $36,24 \%$ \\
\hline Institución Educativa Fiscomisional & $32,14 \%$ \\
\hline Institución Educativa Particular & $31,62 \%$ \\
\hline TOTAL & $100,00 \%$ \\
\hline
\end{tabular}

Fuente: Encuesta La Taxonomía de Bloom para la Era Digital

Según los datos de la Tabla 7 se puede analizar que la actividad digital de la Taxonomía de Bloom en la habilidad Comprender y en la actividad de Mostrar y Contar, donde Contar es mencionar o hacer un resumen con sus propias palabras de forma general lo que ha ocurrido y Mostrar es describir mediante palabras más específicas que evocan imágenes concretas en la mente del lector (Amor Pérez y Delgado Huelva, 2012; Anderson y Krathwohl, 2001; Churches, 2009), quienes mayormente destacan en esta habilidad y actividad es la Institución Fiscal con un 36.24\%, seguido de la Institución Fiscomisional con un 32.14\% y finalmente la Institución Particular con $31.62 \%$.

En consecuencia, se observa que los docentes de las Instituciones Fiscomisional y Particular son los que menos utilizaron la actividad digital Mostrar y Contar, mientras que los docentes de la Institución Fiscal emplean mayormente esta habilidad y actividad utilizando herramientas de audio como grabador de sonidos de Audacity y herramientas de podcasting, herramientas de video, Mapa mental, lo que ha permitido que los estudiantes pueden comprender mejor y corroborando nuevamente con Churches (2009), quién fue él que actualizó la Taxonomía de Bloom que atiende a los nuevos aprendizajes mediados por las TIC y la puso en relación con las nuevas realidades de la Era Digital, complementando cada categoría con verbos y herramientas del mundo digital, todo ello con el fin de desarrollar habilidades.

\subsection{Actividad Digital para Comprender: Listar}

Tabla 8

Actividad digital para Comprender: Listar

\begin{tabular}{|l|r|}
\hline \multicolumn{2}{|l|}{ Actividad Digital en la Habilidad Comprender: Actividad } \\
Listar \\
\hline Institución Educativa Fiscal & $8,47 \%$ \\
\hline Institución Educativa Fiscomisional & $26,42 \%$ \\
\hline Institución Educativa Particular & $65,11 \%$ \\
\hline TOTAL & $100,00 \%$ \\
\hline
\end{tabular}

Fuente: Encuesta La Taxonomía de Bloom para la Era Digital

La Tabla 8 trata sobre la actividad digital de la Taxonomía de Bloom en la habilidad Comprender y en la actividad de Listar; esta actividad consiste en realizar una serie ordenada de datos o palabras (Amor Pérez y Delgado 
Huelva, 2012; Anderson y Krathwohl, 2001; Churches, 2009), se puede deducir que esta habilidad y actividad se ha visto utilizada en su mayoría en la Institución Particular con un 65,11\%, los docentes de la Institución Fiscomisional utilizan en un $26.42 \%$ y en menor porcentaje los docentes de la Institución Fiscal con un $8.47 \%$; esto permite conocer que los docentes de la Institución Particular emplean la habilidad de Listar en mayor proporción utilizando Procesador de Texto, Mapas Conceptuales, fomentando el desarrollo de la comprensión por parte de los estudiantes. Es aquí donde la Taxonomía de Bloom ordena un conjunto de habilidades intelectuales desde un nivel simple hacia la complejidad, el cual además de ser jerárquico es acumulativo, puesto que para avanzar un nivel el estudiante tiene que dominar el nivel anterior (Barcia Menéndez, Carvajal Zambrano, Barcia Menéndez, y Sánchez García, 2017).

\section{Conclusiones}

En conclusión, se logró determinar que la actividad digital con mayor aplicabilidad docente de la Taxonomía de Bloom fue la actividad Listar con un alto porcentaje en la Institución Particular la cual permite a mayor escala potenciar el nivel de comprensión en los estudiantes de Octavo, Noveno y Décimo Grado de Educación General Básica (EGB). durante el año lectivo 2019 - 2020 en el sector urbano de la ciudad de Loja - Ecuador.

La institución Particular alcanzó el mayor porcentaje de utilización de la Taxonomía de Bloom, cuenta con docentes que consideran la utilización de las actividades digitales de listar, explicar y resumir, de tal forma que el ámbito educativo se encuentra enfocado en el desarrollo del proceso de enseñanza y aprendizaje, permitiéndose enriquecer y diversificar aún más el ámbito educativo para lograr una mayor formación de los estudiantes. Por el contrario, los docentes de las instituciones Fiscal y Fiscomisional se evidencia que no cuentan con un mismo nivel académico, provocado por la falta de recursos ante la gran demanda de estudiantes y las escasas capacitaciones de los docentes que dificulta mejorar el nivel de comprensión en la Era Digital.

Se contempla que en la Institución Educativa Fiscal existe una gran brecha digital referenciado en la Taxonomía de Bloom, presentándose de tal forma un bajo nivel de compresión por parte de los estudiantes, lo cuales ante ello también se encuentra como factor detonante directo la falta de recursos y la sobrecarga laboral para los docentes. La institución fiscomisional se encuentra en un ámbito intermedio de la de aplicación docente en las Actividades Digitales para Comprender según el marco referencial de la Taxonomía de Bloom.

\section{Referencias bibliográficas}

Aliaga, S. (2012). Taxonomía de Bloom. Universidad Cesar Vallejo, 4. Obtenido de https://santiagowalteraliagaolivera.files.wordpress.com/2012/03/4-taxonomia-de-bloom1.pdf

Anderson, L.W., and D. Krathwohl (Eds.) (2001). A Taxonomy for Learning, Teaching and Assessing: a Revision of Bloom's Taxonomy of Educational Objectives. Longman, New York.

Barcia Menéndez, J. j., Carvajal Zambrano, B. T., Barcia Menéndez, C. R., y Sánchez García, E. G. (2017). El proceso de enseñanza-aprendizaje en la Educación Superior. La dinámica de los componentes didácticos en sistemas de clases. Manabí, Ecuador: Mar Abierto.

Bloom, B. (1990). Taxonomía de los objetivos de la educación. Buenos Aires: El Ateneo.

Careiro, M. (2019). Recreando la Taxonomía de Bloom para niños artistas. ArtsEduca. Obtenido de https://dialnet.unirioja.es/servlet/articulo?codigo=7165000

Cazau, P. (2006). Introducción a la investigación en ciencias sociales. Obtenido de http://alcazaba.unex.es/asg/400758/MATERIALES/INTRODUCCI\%C3\%93N\%20A\%20LA\%20INVESTIGACI\%C 3\%93N\%20EN\%20CC.SS..pdf 
CEAACES. (2013). Modelo para la Evaluación de las Carreras Presenciales y Semi-presenciales de las Universidades y Escuelas Politécnicas del Ecuador. Quito. Obtenido de http://dspace.ucuenca.edu.ec/bitstream/123456789/22922/1/1.pdf

Chero, H. (2009). Taxonomía de Bloom para la ERA DIGITAL (Andrew Churches). Obtenido de https://reddolac.org/m/blogpost?id=2709308\%3ABlogPost\%3A12107\&maxDate=2013-0305T08\%3A35\%3A55.312Z

Churches, A. (01 de octubre de 2009). Taxonomía de Bloom para la Era Digital. Recuperado el 06 de abril de 2020, de Eduteka: http://eduteka.icesi.edu.co/articulos/TaxonomiaBloomDigital

Der Bijl, B. (2015). La evaluación de carreras universitarias en el Ecuador ¿Desde qué concepción de educación? Universidad de Cuenca, 9-22. Obtenido de http://dspace.ucuenca.edu.ec/bitstream/123456789/22922/1/1.pdf

Eduteka. (2010). La Taxonomía de Bloom y sus dos actualizaciones . Obtenido de https://www.academia.edu/4265277/LA_TAXONOMIA_DE_BLOOM

Hallak , J., Ordoñez , V., Maclean , R., Irvine , J., Bordia , A., Bray, M., . . E Eisner, E. W. (2000). La Educación en Asia. Perspectivas, 30(3), 423-432. Obtenido de https://unesdoc.unesco.org/ark:/48223/pf0000123139_spa/PDF/123140spao.pdf.multi.nameddest=1231 39

Hyder, I., y Bhamani, S. (2016). Bloom's Taxonomy (Cognitive Domain) in Higher Education Settings: Reflection Brief. Education and Educational Development, 3(2), 14. Obtenido de https://www.researchgate.net/publication/311614702_Bloom\%27s_Taxonomy_Cognitive_Domain_in_Hig her_Education_Settings_Reflection_Brief

Forehand, M. (2011). Bloom's Taxonomy. Obtenido de https://www.d41.org/cms/lib/IL01904672/Centricity/Domain/422/BloomsTaxonomy.pdf

Fuller, U., Johnson, C., Ahoniemi , T., Cukierman , D., Losada, I., Jackova, J., . . Thompson , E. (2007). Developing a Computer Science-specific. 152-170. Obtenido de https://www.cs.kent.ac.uk/pubs/2007/2798/content.pdf

Geoff, I. (1996). Bloom's taxonomy of educational objective. Obtenido de https://kaneb.nd.edu/assets/137952/bloom.pdf

Hargreaves, A. (2003). Enseñar en la sociedad del conocimiento. Barcelona. Octaedro.

Hernández Sampieri, R., Fernández Collado, C., y Baptista Lucio, M. d. (2014). Metodología de la Investigación (Sexta ed.). México: McGRAW-HILL. doi: 978-1-4562-2396-0

Hyder , I., y Bhamani, S. (2016). Bloom's Taxonomy (Cognitive Domain) in Higher Education Settings: Reflection Brief. Education and Educational Development, 3(2). Obtenido de https://www.researchgate.net/publication/311614702_Bloom's_Taxonomy_Cognitive_Domain_in_Higher _Education_Settings_Reflection_Brief

López, J. (2014). Ta Taxonomía de Bloom y sus actualizaciones. Eduteka. Obtenido de http://revistapedagogicanuevaescuela.blogspot.com/2014/09/taxonomia-de-bloom-y-susactualizaciones.html 
Masapanta, S., y Velázquez, J. (2017). Primeros pasos para una mejora en el uso de la taxonomía de Bloom en la enseñanza de la informática. Iberoamericana de Informática Educativa, 1-12.

Ministerio de Educación. (2012). Estándares de calidad educativa Aprendizaje, Gestión Escolar, Desempeño Profesional e Infraestructura. Obtenido de https://educacion.gob.ec/wpcontent/uploads/downloads/2013/03/estandares_2012.pdf

Ministerio de Educación. (2017). Gestión Escolar, Desempeño Profesional Directivo y Desempeño Profesional Docente. Obtenido de https://educacion.gob.ec/wp-content/uploads/downloads/2017/12/Manual-parala-implementacion-de-los-estandares-de-calidad-educativa.pdf

Woolfolk, A. (2010). Psicología educativa (11a. edición ed.). México: Pearson Educación 


\section{Anexos}

La Taxonomia de Bloom para la Era Digital: Actividades digitales docentes en Octavo, Noveno y Décimo Grado de Educación General Básica (EGB) en la Habilidad de "Comprender"

Estimado (a) docente:

Solicitando gentilmente $\mathrm{y}$ con mucho respeto, que complete la información del presente cuestionario con el mayor detalle posible; permitiendo potenciar el desarrollo de la educación a nivel institucional. La información suministrada será manejada confidencialmente, sin evidenciar casos particulares.

1. INFORMACIÓN GENERAL:

a. Tipo de Institución Educativa

\begin{tabular}{|l|l|}
\hline FISCAL & \\
\hline FISCOMISIONAL & \\
\hline PARTICULAR & \\
\hline
\end{tabular}

2. ACTIVIDADES DIGITALES DOCENTES EN OCTAVO, NOVENO Y DECIMO GRADO DE EDUCACIÓN GENERAL BÁSICA (EGB) EN LA HABILIDAD DE "COMPRENDER": Las actividades para llevarse a cabo para que los estudiantes comprendan son las siguientes (Amor Pérez y Delgado Huelva, 2012; Churches, 2009):

\begin{tabular}{|c|c|c|}
\hline \multicolumn{3}{|c|}{ Actividad Digital para la Habilidad Comprender: Actividad Resumir } \\
\hline DEFINICIÓN & \multicolumn{2}{|c|}{ Alternativas de Respuesta } \\
\hline $\begin{array}{l}\text { Se puede efectuar a través de procesadores de } \\
\text { texto, mapas conceptuales, publicar en la } \\
\text { Web, Auto publicaciones simples (diarios en } \\
\text { blogs y paginas sencillas de elaboración } \\
\text { colaborativa de documentos). }\end{array}$ & SI & NO \\
\hline \multicolumn{3}{|c|}{ Actividad Digital para la Habilidad Comprender: Actividad Recolectar } \\
\hline DEFINICIÓN & \multicolumn{2}{|c|}{ Alternativas de Respuesta } \\
\hline \multirow{2}{*}{$\begin{array}{l}\text { Procesador de texto, mapa mental, publicar en } \\
\text { la Web, diarios en blogs y páginas sencillas de } \\
\text { construcción colaborativa de documentos, } \\
\text { Wiki. }\end{array}$} & SI & NO \\
\hline & & \\
\hline \multicolumn{3}{|c|}{ Actividad Digital para la Habilidad Comprender: Actividad Explicar } \\
\hline DEFINICIÓN & \multicolumn{2}{|c|}{ Alternativas de Respuesta } \\
\hline $\begin{array}{l}\text { Procesador de texto, mapas conceptuales, } \\
\text { publicar en la Web, Auto publicaciones } \\
\text { simples, Wikis. }\end{array}$ & SI & NO \\
\hline \multicolumn{3}{|c|}{ Actividad Digital para la Habilidad Comprender: Actividad Mostrar y Contar } \\
\hline DEFINICIÓN & \multicolumn{2}{|c|}{ Alternativas de Respuesta } \\
\hline \multirow{2}{*}{$\begin{array}{l}\text { Procesador de texto, presentaciones en linea } \\
\text { o locales, gráficas, herramientas de audio, } \\
\text { herramientas de video, mapa mental. }\end{array}$} & SI & NO \\
\hline & & \\
\hline \multicolumn{3}{|c|}{ Actividad Digital para la Habilidad Comprender: Actividad Listar } \\
\hline DEFINICIÓN & \multicolumn{2}{|c|}{ Alternativas de Respuesta } \\
\hline \multirow{2}{*}{ Procesador de texto, mapas conceptuales. } & SI & NO \\
\hline & & \\
\hline
\end{tabular}


Esta obra está bajo una Licencia Creative Commons Attribución-NoCommercial 4.0 International

\section{(cc) BY-NC}

